\title{
New layers to recombination patterns
}

\section{revisions are \\ needed to \\ models for \\ detecting and \\ characterizing \\ the effects of \\ selection

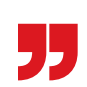

Photodisc

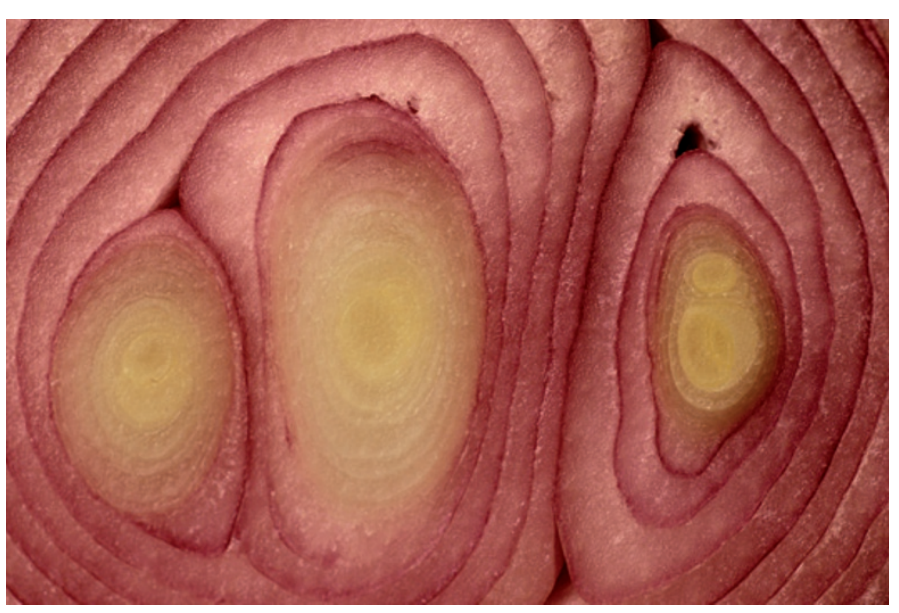

genomes that could distinguish between the parental $D$. melanogaster strains and then identifying recombination events from switches in parental origin. Altogether, the products of 5,860 female meioses were characterized, and more than 106,000 recombination events - including both crossing over and gene conversion - were mapped at a resolution down to $2 \mathrm{~kb}$.

The overall picture from further analysis of these data is one of variation in recombination rates on several levels. The detection of more than 32,500 crossing-over events - which the authors believe to be an exhaustive survey - allowed the generation of a high-resolution genome-wide map of crossing over in D. melanogaster. This map revealed widespread variation in crossingover rates both across large physical distances and on a finer scale. For example, adjacent regions of $100 \mathrm{~kb}$ showed 15-20-fold differences in the recombination rate. Both hotspots and coldspots of recombination were identified. Importantly, a large amount of intraspecific variation in crossing over was also seen, much of which is associated with hotspots that occur at a low frequency.

Comeron and colleagues also generated maps of gene conversion consisting of a total of 74,453 events that are distributed more uniformly across the genome than are crossing-over events. Importantly, the data show that gene conversion occurs more frequently than crossing over: around $83 \%$ of recombination events involve gene conversion, and gene conversion even occurs in regions in which there is no crossing over.

Another topic into which these data provide new insight is the sequence motifs involved in recombination in D. melanogaster. The findings contrast to the situation in mammals such as mice and humans, in which recombination hotspots are highly localized and involve a limited set of sequence motifs. Instead, the authors identified a much larger number of motifs associated with D. melanogaster recombination events spread over larger genomic regions. The findings indicate a more probabilistic control of recombination in the fruitfly than in mammals.

Finally, the new maps show that recombination in $D$. melanogaster occurs significantly more often in genic compared with intergenic regions. The specific locations of recombination events within genes suggest that increased chromatin accessibility due to transcription may be involved in determining the sites of double-strand breaks.

The findings presented in this study provide new avenues for understanding how recombination patterns arise. They also suggest that revisions are needed to models for detecting and characterizing the effects of selection. For example, it is clear that variation in recombination rates within species needs to be taken into account in such models, as does the rate of gene conversion, given that most current models are based on crossing-over frequencies alone. Louisa Flintoft

ORIGINAL RESEARCH PAPER Comeron, J. M., Ratnappan, R. \& Bailin, S. The many landscapes of recombination in Drosophila melanogaster. PLoS Genet. 8, e1002905 (2012) 